\title{
Integration of National AIDS Control Program and Pharmacovigilance Program of India-Antiretrovi- ral Drugs safety coactions in India

\author{
Case Report
}

Kalaiselvan $\mathrm{V}^{1^{*}}$, Prasad $\mathrm{T}^{1}$, Vivek $\mathrm{D}^{1}$, Gyanendra Nath Singh ${ }^{1}$, Rathore A.S $\mathrm{S}^{2}$, Rewari B.B ${ }^{2}$

${ }^{1}$ National Coordination Centre-Pharmacovigilance Program of India, Indian Pharmacopoeia Commission, Ministry of Health \& Family Welfare, India. ${ }^{2}$ National AIDS Control Organization, Ministry of Health \& Family Welfare, India.

\section{*Corresponding Author:}

Kalaiselvan V,

National Coordination Centre-Pharmacovigilance Program of India, Indian Pharmacopoeia Commission, Ministry of Health \& Family Welfare, Sector-23, Raj Nagar, Ghaziabad, Uttar Pradesh-201002, India.

E-mail: vivekarts@rediffmail.com

Received: April 24, 2015

Accepted: May 25, 2015

Published: May 27, 2015

Citation: Kalaiselvan V et al., (2015) Integration of National AIDS Control Program and Pharmacovigilance Program of India-Antiretroviral Drugs safety coactions in India. Int J AIDS Res. 02(4), 37-38. doi: http://dx.doi.org/10.19070/2379-1586-150008

Copyright: Kalaiselvan $\mathbf{V}^{\circ}$ 2015. This is an open-access article distributed under the terms of the Creative Commons Attribution License, which permits unrestricted use, distribution and reproduction in any medium, provided the original author and source are credited.

This letter describes the initiatives taken by Indian Pharmacopoeia Commission (IPC) in monitoring safety of Anti-Retroviral Therapy (ART) drugs $[1,2,3]$. The Collaboration between $\mathrm{Na}-$ tional AIDS control organization (NACO) \& Pharmacovigilance Program of India (PvPI) is aimed to work with periphery ART centres to monitor the safety of ART drugs \& also to initiate the Cohort Event Monitoring (CEM) thus to perform the benefitrisk assessment of ART drugs, however there are some technical \& operational issues in implementing the program \& identifying the risk related to ART drugs. Pharmacovigilance is the science and activities relating to detection, assessment, understanding and prevention adverse effects or any other possible drug related problems [4]. It is an integral part of every public health program that uses medicines, optimizing their safety is paramount (World Health Organization-Uppsala Monitoring Centre: http:// www.who.int/medicines/areas/quality_safety/safety_efficacy/ Pharmacovigilance_B.pdf) Failure to monitor, understand and manage these events can result in poor adherence and treatment failure and can reduces confidence of Public (UNAIDS I World Health Organization: http://www.who.int/hiv/pub/tech_guidance_pharma.pdf). Therefore to ensure safe use of medicines and promote patients safety, Ministry of Health and Family Welfare, Government of India, launched a nationwide PvPI to monitor the safety of medicines. IPC functions as National Coordination Centre (NCC) for PvPI. To monitor adverse drug reactions (ADRs), and report to NCC, ADRs monitoring centres (AMCs) which are the medical institutions or corporate hospitals have been set up across the country [5]. IPC is an autonomous institution of the Ministry of Health and Family Welfare, Government of India and functioning as NCC for PvPI which collaborates with the World Health Organization (WHO) -Uppsala Monitoring Centre (UMC), Sweden.

India has the third largest number of people living with HIV in the world, 2.1 million at the end of 2013 and accounts for about 4 out of 10 people living with HIV in the region, In India, the numbers of new HIV infections declined by 19 percent, yet it still accounted for 38 percent of all new HIV infections in the region. India recorded a 38 per cent decline in AIDS related deaths between 2005 and 2013. During this period, there was a major scale up of access to HIV treatment. At the end of 2013, more than 700,000 people were on ART, the second largest number of people on treatment in any single country (The Gap Report, UNAIDS:http://www.unaids.org/sites/default/files/en/media/ unaids/contentassets/documents/unaidspublication/2014/UNAIDS_Gap_report_en.pdf). During last five years there were 11 ART fixed drug combinations (FDCs) were approved for manufacturing in India.

With the establishment of ART in 2004, the national treatment program has scaled-up provision of ART to about 796,269 people (National AIDS Control Organization: http:/ / www.naco.gov. in/upload/2014). In order to make treatment more accessible, ART centres are located in tertiary/district hospitals and medical colleges [6]. For many of the ART medicines, their outcomes on long term use are still not known. Lack of information may lead to loss of patient confidence that may lead to suboptimal levels of adherence to the treatment.

To ensure the safety of ART medicines used in the program, IPC, NCC-PvPI and NACO formally agreed to collaborate on $15^{\text {th }}$ September 2014, for setting up systems and processes for reporting, analysis and monitoring of ADR due to ART medicines used in National AIDS Control program (NACP). In the first phase, 30 ART Centres were identified from the existing AMCs under PvPI and health care workers from ART centres and healthcare professionals from existing AMCs have been trained on basic concepts, terminologies and Standard Operating Procedures and also provide hands on training on ADR form filling, Vigiflow data entry and various other aspects of PvPI. All these ART centres have been provided with accessibility to VigiFlow for ICSR management system.

A health care professional plays a vital role in ADR reporting by counselling patients, thereafter the Technical Associate (TA) is responsible for collecting ADR reports and follows up for the reported ADRs. All the scrutinized and signed ADR reports are entered into the Vigiflow by the TA. Every report then comes 
under central assessment to NCC for quality review of ICSRs.

There is a need to bridge the gap between AMCs and ART centres in monitoring and reporting ADRs with the use of ART drugs. This will be addressed by regular interactive session and meetings for better coactions and to harmonize the process of ADR reporting.

Integration of PvPI with NACP gives a scope of performing CEM for the earliest possible recognition of new ADRs of ART drugs including interactions, benefit-risk assessment of different ART regimens or products \& evidence based regulatory action. In CEM, all adverse events occurring to a patient taking ART are collected regardless of the causality or relationship with the ART drugs.

The advantages of CEM over spontaneous reporting include the ability to produce rates, rapid results, and early detection of signals, fewer missing data and less reporting bias [7]. However, CEM requires more resources than spontaneous reporting. So based upon the performance of respective ART centres, NCCPvPI would initiate active pharmacovigilance surveillance/CEM for newly launched FDCs in these ART centres on performance priority basis.

For many of the ART drugs their outcomes on long term use are still not known, since this is a national level initiation, such events may be observed. Also the public confidence will be enhanced about the safety of ART drugs used in the NACP. PvPI is now an integral part of NACP in order to optimize the use of scarce health resources and prevent potential tragedies.

\section{Acknowledgement}

NCC-PvPI, IPC sincerely acknowledge the higher officials of Ministry of Health \& Family Welfare, Government of India and Dr. Madhur Gupta, Technical Officer (Pharmaceuticals)-WHO, Country Office (India) for their support and guidance for the collaboration.

\section{References}

[1]. Kalaiselvan V, Prasad T, Akansha B, Surinder S, Gyanendra Nath S (2014) Adverse drug reactions reporting culture in Pharmacovigilance program of India. Indian J Med Res 140(4): 563-64.

[2]. Kalaiselvan V, Kumar R, Prasad T, Traipathi A, Gyanendra Nath S (2015) Status of documentation grading and completeness score for Indian individual case safety reports. Indian J of Phar 47(3): 325-27.

[3]. Kalaiselvan V, Prabhakar M, Gyanendra Nath S (2014) Helpline facility to assist reporting of adverse drug reactions in India. WHO South-East Asia. J of Public Health 3(2): 194.

[4]. Meyboom RHB, Egberts ACG, Gribnau FWJ, Hekster YA (1999) Pharmacovigilance in perspective. Drug Safety 21(6): 429-47.

[5]. Kalaiselvan V, Prasad T, Abishank S (2014) Current Status of Adverse Drug Reactions Monitoring Centres under Pharmacovigilance program of India. Indian Journal of Pharmacy Practice 7(3): 19-22.

[6]. Salma F, Suresh S, Bharat R (2012) Decentralizing treatment services with link ART centres- experience from Karnataka, South India. Retrovirology 9(Suppl 1): 82.

[7]. Shanthi N, Chris D, Dennis F, Sten O (2013) WHO Strategy for Collecting Safety Data in Public Health Programs, Complementing Spontaneous Reporting Systems. Drug Safety 36(2): 75-81. 